\title{
MÁS DE DOS DÉCADAS EN CUIDADOS \\ PALIATIVOS EN COSTA RICA
}

\author{
Javier A. Rojas Elizondo*
}

\section{RESUMEN}

Este artículo presenta una síntesis sobre el desarrollo de los cuidados paliativos en Costa Rica, que se articulan por medio del Consejo Nacional de Cuidados Paliativos, institución ligada al Ministerio de Salud Pública. Se concluye que es esencial la coordinación de distintos organismos que trabajan por el consenso en la atención mediante el establecimiento de normas, protocolos y guías clínicas orientadores con lineamientos claros a los profesionales de salud en la atención al final de la vida.

Palabras Clave: cuidados paliativos, paciente en fase terminal, Costa Rica.

MORE THAN TWO DECADES

OF PALLIATIVE CARE IN COSTA RICA

\section{Abstract}

This article presents a synthesis on the development of palliative care in Costa Rica, articulated through the National Council for Palliative Care, an institution linked to the Ministry of Public Health. We conclude that the coordination among different organisms that work on behalf of consensus in care is essential to establish norms, protocols, and clinical guidelines for health professionals in end-of-life care.

KeYwords: palliative care, terminal patient, Costa Rica. 
Para los compañeros y compañeras de la Sociedad Interamericana de Tanatología es fundamental tener una visión clara vinculada con la realidad del sistema de salud de Costa Rica, toda vez que el desarrollo de los cuidados paliativos está íntimamente relacionado con el estudio y evolución de los mismos.

Costa Rica es una nación pequeña situada en Centroamérica, con una superficie de $51100 \mathrm{~km}^{2}$, es un destino turístico para visitantes de todo el mundo. Tiene una variedad de especies animales y vegetales propios de la zona tropical que responden a la ubicación del país. Costa Rica por su posición significó un puente biológico y cultural que permitió el encuentro de especies forestales y animales, así como de culturas del norte y del sur del continente. La diversidad de flora y fauna es de las más altas del mundo. Su posición ístmica le permitió la migración de especies del norte y el sur de América. Casi el 30\% del territorio está protegido bajo diversas formas de conservación (FAO, 2000).

La cultura y gentilicio costarricense son una mezcla de razas que coexisten en el país. La influencia principal es la europea, que se demuestra en aspectos como el idioma y la arquitectura. La influencia indígena es menos visible, pero está presente en algunos platos típicos y el desarrollo artístico. Una influencia más reciente es la que viene de Estados Unidos, que se observa en muchas áreas, desde las carteleras publicitarias, costumbres y cadenas de comida que abundan en los pueblos.

Un aspecto importante de la herencia cultural costarricense es su amor por la paz y la democracia. Los «ticos», como se les conoce a los costarricenses, tratan de destacar que el país es la excepción de América Latina, ya que desde hace mucho tiempo la política es dominada por el sistema democrático y no por dictadores. Los ticos se enorgullecen de tener más de un siglo de tradición democrática y más de 50 años sin ejército. Éste fue abolido en 1948, y el dinero que ahorra el país al no tener fuerzas armadas se invierte en mejorar el nivel de salud de los costarricenses, lo que ayuda para la paz social, que hace de Costa Rica un lugar considerado como agradable.

Costa Rica tiene un contexto de salud en las Américas que puede percibirse como evidentemente privilegiado; no obstante, si se compara con países desarrollados, indudablemente es mucho lo que nos falta por hacer y más aún lo que necesitamos aprender.

Lo mencionado ubica al país entre los doce del mundo que más invierten en salud y el número uno que más invierte a nivel de América Latina, según la Organización para la Cooperación y el Desarrollo Económicos (OCDE). El sistema de seguridad social del país, a cargo de la Caja Costarricense de Seguro Social, es de tipo universal para toda la población.

La salud de una comunidad estriba en parte de los servicios de salud constituidos, pero en una medida mucho mayor depende de las condiciones del ambiente, del tipo de vida y trabajo que la persona posea, de los alimentos que se consuman, de

* PhD. Dirección de correspondencia javierrojas1@yahoo.com. 
las condiciones de la vivienda, de la pureza del agua, de cómo se emplea el tiempo libre, de los hábitos de reproducción, del nivel de salud mental, entre muchos otros. Es decir, la integridad física y mental de las personas está relacionada con la forma de vida y con una responsabilidad individual y social de la sociedad.

En nuestro país, en las décadas anteriores, un sostenido aumento del ingreso per cápita y de los salarios en general coincidió con una clara mejoría de los indicadores de salud, no quiere esto indicar una relación directa de causa y efecto, sino simplemente mostrar una relación que coincide razonablemente con la tesis de que el desarrollo económico y social favorece el mejoramiento de la salud (Mohs, 1983). Desafortunadamente, tal situación de prosperidad general desapareció muy rápidamente, por lo que el sistema entró en una crisis organizacional y financiera que ha limitado la inversión en salud y que, a pesar de las reformas sectoriales, persiste en la actualidad. Hoy existe preocupación sobre la sustentabilidad financiera del sistema a largo plazo. Con todo eso el país muestra un considerable aumento de la esperanza de vida al nacer, que de alguna manera es paradójico en tanto que se tienen mayor cantidad de enfermedades crónicas y cáncer con menos recursos económicos para tratarlas adecuadamente.

El desarrollo de los cuidados paliativos y, en un sentido más amplio, del sistema nacional de salud está relacionado con el nivel de vida de cada sociedad. En general, a mayor bienestar económico la inversión en gasto sanitario y -más concretamente- en cuidados paliativos parece aumentar (Pastrana, 2013).

La medicina del dolor y cuidados paliativos de Costa Rica se trabaja en red por medio de la seguridad social, desde el primer hasta el tercer nivel de atención. La red se encuentra articulada por medio del Consejo Nacional de Cuidados Paliativos, que está ligado al Ministerio de Salud Pública.

El país cuenta con el Centro Nacional del Control del Dolor y Cuidados Paliativos, con 51 unidades a lo largo y ancho del país. Al ańo, el centro atiende alrededor 11700 pacientes y las otras unidades en el resto del país atienden cerca de 12000 pacientes al año (CNCD y CP, 2020). La intención es contribuir a desarrollar una sólida Red Nacional de Atención del Dolor y de Cuidados Paliativos, que, trabajando articuladamente con el Consejo Nacional de Cuidados Paliativos adscrito al Ministerio de Salud y con las respectivas asociaciones de especialistas del Colegio de Médicos y Cirujanos de Costa Rica, contribuya a regular la actividad tanto en el sector público como en el privado. Se están desarrollando normativas de atención, consensos, protocolos y guías clínicas que orienten adecuadamente y con lineamientos claros a profesionales de salud en general, a la hora de atender a estos pacientes.

A nivel histórico la década de los noventa representó el período de florecimiento de los cuidados paliativos en Costa Rica, donde surgieron diferentes centros encargados de esta labor, pertenecientes tanto a la CCSS como a organizaciones no gubernamentales (ONG) (Brenes, M., Brenes, J., \& Núñez, C, 2013).

A finales de la década de los ochenta se funda la primera Unidad Pediátrica en Costa Rica y en América Latina que brinda atención domiciliaria y hospitalaria a los niños del país. De acuerdo a lo recopilado por Brenes (2013), los cuidados paliativos tienen su origen a la luz del sufrimiento de una paciente, ya que las far- 
macias se negaban a despachar las dosis de morfina prescritas. Esto, asociado a la escasez del fármaco en el país y al desconocimiento en cuanto a la dosis, que en esa época era considerada poco científica por algunos profesionales que no se encontraban actualizados en terapia del dolor y el cuidado paliativo (Brenes, M., Brenes, J., \& Núñez, C, 2013). Lo anterior motivó algunas querellas legales que concluyeron en la protección del Estado en cuanto al derecho de «morir con dignidad y sin dolor» para toda la población.

Poco tiempo después se crea la primera Clínica del Control del Dolor y Cuidados Paliativos del país, ya que los otros centros que se habían establecido hasta el momento se orientaban al cuidado paliativo. En dicha clínica se brindaba la atención de los pacientes a nivel de consulta externa y hospitalización; posteriormente, debido a que ya los pacientes no se podían presentar a consulta médica debido a su condición, se inicia la visita domiciliar (Brenes, M., Brenes, J., \& Núñez, C, 2013). Dentro de la atención que brinda esta unidad se encuentran personas con enfermedades degenerativas que afectan al corazón, los pulmones, el hígado, los rińones o el cerebro, o enfermedades crónicas o potencialmente mortales, como la infección por VIH y la tuberculosis farmacorresistente. Algunos años después esta Clínica del Dolor cambió su nombre a Centro Nacional de Control del Dolor y Cuidado Paliativo y fue declarada como la encargada técnica, asesora en su especialidad, en el ámbito institucional en medicina del dolor, apoyo emocional y atención psicosocial al enfermo en fase terminal.

La creación de estas instituciones brindó respuesta a una gran cantidad de necesidades que no se habían tomado en consideración. También mitigó las privaciones que tenía el país en el apoyo a personas y grupos familiares que tenían grandes necesidades en materia de apoyo en el progreso de la enfermedad. Es a partir de estos momentos cuando surge una lucha de los hospitales y comunidades para crear sus unidades de Cuidado Paliativo.

Uno de los avances más considerables en materia de política pública fue la promulgación de la Ley N. ${ }^{\circ} 7756$ del 20 de marzo de 1998, con el fin de brindar licencia al trabajador asalariado activo que fuera designado como beneficiario responsable de cuidar a un paciente en fase terminal, sin mediar retribución alguna entre el paciente y la persona responsable de cuidarlo; el responsable podrá ser familiar u otra persona que por su vínculo afectivo y responsabilidad, se estime cumpla con la misión encomendada.

La "Ley de Beneficios para los Responsables de Pacientes en Fase Terminal» tenía por objeto que las personas, que habían sido diagnosticadas con una enfermedad en fase terminal -y que tenían una expectativa de vida igual o menor a seis meses, según lo disponía el artículo 3, de la Ley- pudieran designar a una persona, sea un familiar o una persona con quien tuvieran un vínculo afectivo, que pueda cuidarlos. La posibilidad de designar un cuidador responsable de una persona con una enfermedad en fase terminal, tiene su fundamento en la dignidad inherente de la persona humana. De allí que la Ley señala, expresamente, que la condición del «Cuidador Responsable» no admite la posibilidad de que el paciente u otra persona retribuya al cuidador de ninguna forma. Empero, la Ley de Responsables de Pacientes Terminales establece que las personas designadas para ser cuidadores res- 
ponsables podrán disfrutar de una licencia laboral y de un subsidio, durante ese tiempo de licencia.

La aplicación de los beneficios de la ley propició el mejoramiento de la calidad de atención de las personas portadoras de enfermedades terminales y permitió que los cuidadores disfrutaran de licencias que les permitieron estar al lado de su familia. En los últimos años en Costa Rica las políticas nacionales de salud han avanzado en cuanto a leyes que preservan y ayudan a aquellas personas que se encuentran en estado terminal y a sus cuidadores más próximos, lo cual ha sido un aporte al progreso del país.

En la actualidad se podría decir que toda la población nacional se beneficia de este avance en la salud, donde ahora no solo la curación es una necesidad, sino también el apoyo continuo en los últimos días de la vida.

En los primeros años de esta década dos universidades costarricenses inician maestrías interdisciplinarias en Cuidado Paliativo. Con estos programas se crea la primera oferta académica formal universitaria en Cuidado Paliativos en Centroamérica (Brenes, M., Brenes, J., \& Núñez, C, 2013). Poco tiempo después el Colegio de Médicos de Costa Rica inicia el proceso de reconocimiento de la especialidad en Cuidados Paliativos a nivel médico.

Otra de las actividades notables que se han realizado ha sido contribuir con la formación permanente y la capacitación de profesionales de salud, para garantizar la buena atención a largo plazo. En ese proceso, la investigación ha sido determinante para documentar experiencias en este campo tan especializado.

En los últimos años Costa Rica ha destacado como uno de los países que poseen un alto índice de Desarrollo de Cuidado Paliativo (ALCP), según lo indica un estudio de la revista Medicina Paliativa (Pastrana, 2013).

La psicología y otras disciplinas entienden que la colaboración entre los diferentes profesionales es más efectiva cuando las tareas están más claramente definidas y cuando la evaluación respetuosa se establece como norma. Los cuidados paliativos han experimentado un gran desarrollo a lo largo de las últimas décadas; no obstante, falta mucho camino por recorrer, y aún no tienen el apoyo suficiente como para responder de forma oportuna y eficaz a las necesidades crecientes de los pacientes. Cada disciplina debería asumir el desafío de encontrar la manera de potenciar e implementar servicios asistenciales capaces de cumplir con esta demanda cada vez más creciente. 


\section{REFERENCIAS BIBLIOGRÁFICAS}

Brenes, M., Brenes, J. y Núńez, C. (2013). Pasado y presente de los cuidados paliativos en Costa Rica. Revista Médica de Costa Rica y Centroamérica, 70(605), 71-76.

CNCD Y CP (2020). Recuperado https://www.presidencia.go.cr/comunicados/2020/03/centro-nacional-del-dolor-inicia-plan-piloto-para-monitoreo-remoto-a-pacientes/.

FAO (2000). Cambios en la Cobertura Forestal de Costa Rica. Recuperado de http://www.fao.org/ forestry/40021-072df4eef5a6e662193ff1698abb61aa6.pdf (consultado el 13 de junio del 2020).

Jefrry, Garza (30 de noviembre de 2015). «Costa Rica entre los que más invierten en salud». La República. Consultado el 12 de agosto de 2016.

Mohs Villalta, Edgar, 1939- La salud en Costa Rica / Edgar Mohs Villalta. -la. Ed.- San José, C.R.: EUNED, 1983.

Pastrana, T., De Lima, L., Pons, J.J. y Centeno, C. (2013). Atlas de Cuidados Paliativos de Latinoamérica. Edición cartográfica 2013. Houston: IAHPC Press. 